\title{
Increased burden of cardiovascular disease in people with liver disease: unequal geographical variations, risk factors and excess years of life lost
}

\author{
Wai Hoong Chang ${ }^{1 \dagger}$, Stefanie H. Mueller ${ }^{1 \dagger}$, Sheng-Chia Chung ${ }^{1}$, Graham R. Foster ${ }^{2}$ and Alvina G. Lai ${ }^{1 *}$ (i)
}

\begin{abstract}
Background: People with liver disease are at increased risk of developing cardiovascular disease (CVD), however, there has yet been an investigation of incidence burden, risk, and premature mortality across a wide range of liver conditions and cardiovascular outcomes.
\end{abstract}

Methods: We employed population-wide electronic health records (EHRs; from 1998 to 2020) consisting of almost 4 million adults to assess regional variations in disease burden of five liver conditions, alcoholic liver disease (ALD), autoimmune liver disease, chronic hepatitis B infection (HBV), chronic hepatitis C infection (HCV) and NAFLD, in England. We analysed regional differences in incidence rates for 17 manifestations of CVD in people with or without liver disease. The associations between biomarkers and comorbidities and risk of CVD in patients with liver disease were estimated using Cox models. For each liver condition, we estimated excess years of life lost (YLL) attributable to CVD (i.e., difference in YLL between people with or without CVD).

Results: The age-standardised incidence rate for any liver disease was 114.5 per 100,000 person years. The highest incidence was observed in NAFLD (85.5), followed by ALD (24.7), HCV (6.0), HBV (4.1) and autoimmune liver disease (3.7). Regionally, the North West and North East regions consistently exhibited high incidence burden. Age-specific incidence rate analyses revealed that the peak incidence for liver disease of non-viral aetiology is reached in individuals aged 50-59 years. Patients with liver disease had a two-fold higher incidence burden of CVD (2634.6 per 100,000 persons) compared to individuals without liver disease (1339.7 per 100,000 persons). When comparing across liver diseases, atrial fibrillation was the most common initial CVD presentation while hypertrophic cardiomyopathy was the least common. We noted strong positive associations between body mass index and current smoking and risk of CVD. Patients who also had diabetes, hypertension, proteinuric kidney disease, chronic kidney disease, diverticular disease and gastro-oesophageal reflex disorders had a higher risk of CVD, as do patients with low albumin, raised C-reactive protein and raised International Normalized Ratio levels. All types of CVD were associated with shorter life expectancies. When evaluating excess YLLs by age of CVD onset and by liver disease type, differences in YLLs, when comparing across CVD types, were more pronounced at younger ages.

\footnotetext{
*Correspondence: Alvina.lai@ucl.ac.uk

†Wai Hoong Chang and Stefanie H. Mueller contributed equally to this work and share first authorship

${ }^{1}$ Institute of Health Informatics, University College London, London, UK

Full list of author information is available at the end of the article
}

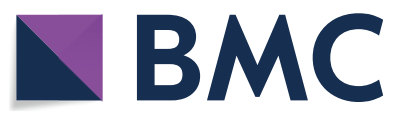

(c) The Author(s) 2021. Open Access This article is licensed under a Creative Commons Attribution 4.0 International License, which permits use, sharing, adaptation, distribution and reproduction in any medium or format, as long as you give appropriate credit to the original author(s) and the source, provide a link to the Creative Commons licence, and indicate if changes were made. The images or other third party material in this article are included in the article's Creative Commons licence, unless indicated otherwise in a credit line to the material. If material is not included in the article's Creative Commons licence and your intended use is not permitted by statutory regulation or exceeds the permitted use, you will need to obtain permission directly from the copyright holder. To view a copy of this licence, visit http://creativecommons.org/licenses/by/4.0/. The Creative Commons Public Domain Dedication waiver (http://creativeco mmons.org/publicdomain/zero/1.0/) applies to the data made available in this article, unless otherwise stated in a credit line to the data. 
Conclusions: We developed a public online app (https://lailab.shinyapps.io/cvd_in_liver_disease/) to showcase results interactively. We provide a blueprint that revealed previously underappreciated clinical factors related to the risk of CVD, which differed in the magnitude of effects across liver diseases. We found significant geographical variations in the burden of liver disease and CVD, highlighting the need to devise local solutions. Targeted policies and regional initiatives addressing underserved communities might help improve equity of access to CVD screening and treatment.

Keywords: Cardiovascular risk, Incidence, Liver disease, Electronic health records, Years of life lost, Geographical variations

\section{Background}

Although cardiovascular disease (CVD) prevention policies have had some success, for the past two decades, they have remained limited in reducing the number of deaths globally with CVD still ranked as the leading cause of death. A large proportion of deaths are attributable to preventable illnesses-most premature deaths from CVD in people younger than 75 are avoidable [1, 2]. As major risk factors for CVD, type 2 diabetes and obesity have received the most attention in policy, public awareness and guidelines. However, despite reported associations between liver disease and cardiovascular risk, liver conditions other than non-alcoholic fatty liver disease (NAFLD) have mostly been overlooked.

Liver disease encompasses a spectrum of conditions including viral hepatitis, NAFLD, steatosis to end-stage cirrhosis. Other causes of liver cirrhosis include autoimmune liver disease and alcoholic liver disease (ALD). The heart has been implicated in the progression of aggravating liver disease, with the liver-heart axis more extensively explored in patients with NAFLD [3]. With a global prevalence of $25 \%$ in the general population [4], the number of individuals living with NAFLD far exceeds the number of individuals with diabetes and obesity [5] combined. NAFLD is characterised by lipid accumulation in the liver and systemic metabolic aberrations, which leads to an increased risk of developing CVD. However, the pathophysiological associations between other liver diseases and CVD remain poorly understood. The World Health Organisation estimated that 250 million individuals are living with chronic hepatitis $\mathrm{B}$ caused by infection with the hepatitis $B$ virus (HBV) where prevalence is the highest in African and Western Pacific regions [6]. An estimated 71 million individuals have chronic hepatitis $\mathrm{C}$ virus ( $\mathrm{HCV}$ ) infection [7] and emerging evidence suggests an association between HCV and CVD, where CVD associated with HCV infection is responsible for the loss of 1.5 million disability-adjusted life-years each year [8]. HBV and HCV transmissions continue to rise in low- and middle-income countries and sustained chronic infection may lead to the development of CVD due to chronic inflammation and metabolic derangements [9].
Additionally, liver disease often co-exists with type 2 diabetes [10] and chronic renal disease [11], both of which are independent cardiovascular risk factors [12].

Current guidelines from the American Association for the Study of Liver Diseases recommended the modification of CVD risk factors in these individuals and the screening of CVD during liver transplant evaluation [13]. Furthermore, since CVD is the most common cause of death in patients with NAFLD, the European Association for the Study of Liver recommends mandatory screening for CVD in individuals with NAFLD [14]. As liver disease progresses, liver-specific risk factors (e.g., raised International Normalised Ratio) and prevalent comorbidities that are independently associated with increased CVD risk may come into play and could result in more severe illness in these individuals. Yet, CVD is often underdiagnosed in patients with liver disease and there are no policies on screening patients with chronic liver disease.

Harnessing an emerging data science opportunity from population-based electronic health records (EHRs), we sought to identify population groups, among patients with liver disease, who might be at high risk for CVD. Employing linked EHRs from primary and secondary care on 4 million individuals, our study aims to address the value, or futility, of targeted monitoring of CVD risk in patients with liver disease. We characterised clinical features of patients with any of the five liver conditions, ALD, autoimmune liver disease, HBV, HCV and NAFLD and explored the associations between risk factors and future risk of 17 of the most common initial cardiovascular presentations. A report on the atlas of variation in risk factors and healthcare for liver disease by the Public Health England found that for the past three decades, there have been limited improvements in mortality rates and incidence of liver disease [15]. Deaths from liver disease have increased by fourfold and progress on earlier diagnosis and better treatment have been slow [16, 17]. For these reasons, we investigated regional variations in liver disease burden and CVD burden to highlight potential gaps in the provision of services, inequality of access and prevention initiatives, drawing attention to regions where improvements are most needed. Specific 
objectives of this study were: (i) to estimate the regional variations in incidence rates for liver disease in England, (ii) to estimate regional variations in incidence rates for CVD in patients with and without liver disease, (iii) to estimate the associations of age, clinical biomarkers, preexisting comorbidities and smoking with risk of initial presentation of CVD and (iv) to estimate excess years of life lost (a marker of pre-mature mortality) based on the age of CVD onset by comparing patients with liver disease who subsequently developed CVD to those who did not.

We provide an open-access online app, with implications for clinical risk assessment and targeted policy on CVD prevention, that shows incidence rates, years of life lost estimations and cause-specific hazard ratios for associations between clinical features and initial presentation of CVD.

\section{Methods}

\section{Study design and data source}

We used linked EHRs from primary and secondary care, which consisted of a population of 3,929,596 adults aged $\geq 30$ years during the study period of 1998-2020. Individuals were followed up until the occurrence of a primary endpoint, death, date of last data collection for the practice, date of administrative censoring (June 2020) or deregistration from the practice (i.e., loss to followup), whichever occurred first. Baseline characteristics were analysed and stratified by liver disease. Information governance approval was obtained from the Medicines Healthcare Regulatory Authority (UK) Independent Scientific Advisory Committee (21_000363) Clinical Practice Research Datalink (CPRD).

\section{Open electronic health record (EHR) definitions of diseases and covariates}

Phenotype definitions of liver disease, CVD, comorbidities and risk factors are available at https://phenotypes. healthdatagateway.org/home/ and have previously been validated [18-23]. Phenotypes for primary care records were generated using Read clinical terminology (version 2). Phenotypes for secondary care records were generated using ICD-10 terms. BMI and smoking were considered as the nearest record to entry within 1 year prior to entry. We examined nine biomarkers; albumin, alanine aminotransferase, aspartate transaminase, bilirubin, C-reactive protein, gamma-glutamyltransferase, International Normalised Ratio (INR), platelets and triglycerides. The primary outcome was the first record of one of the following 17 cardiovascular presentations in data from either primary or secondary care, which were grouped into five CVD categories: (i) coronary heart disease: stable angina, unstable angina, myocardial infarction, heart failure and coronary heart disease unspecified; (ii) strokes and transient ischaemic attack (TIA): ischaemic stroke, stroke unspecified and TIA; (iii) peripheral vascular disease: peripheral arterial disease, pulmonary embolism, venous thromboembolic disease and abdominal aortic aneurysm; (iv) cardiomyopathy: dilated cardiomyopathy, hypertrophic cardiomyopathy and cardiomyopathy unspecified; (v) arrhythmia: atrial fibrillation and sick sinus syndrome. We examined 14 comorbidities and considered records for comorbidities prior to cohort entry (prevalent comorbidities). The comorbidities were diabetes mellitus, complications of diabetes (i.e., diabetic nephropathy, diabetic retinopathy and neurological complications of diabetes), dyslipidaemia, hypertension, jaundice, proteinuric kidney disease, oesophagitis or oesophageal ulcer, proteinuria, chronic kidney disease and gastrointestinal conditions such as Barrett's oesophagus, Crohn's disease, diverticular disease of the intestine, gastro-oesophageal reflux disease and irritable bowel syndrome (Additional file 1 and Additional file 2).

\section{Estimations of age-standardised and age-specific incidence rates}

Age-standardised incidence rates were estimated per 100,000 person-years and based on a 5 -year study period from 01.01.2015 to 31.12.2019. We analysed geographical variations in incidence rates based on CPRD practice region definitions. We retrieved the 2019 population estimates (overall and by age groups) from the Office for National Statistics [24] for age-standardisation of incidence rates. Additionally, we estimated incidence rates by age groups (i.e., age-specific incidence rates) to explore differences across the age groups. Patient years were calculated by age group (30-39, 40-49, 50-59, 60-69, $70-79$ and $\geq 80$ ) using the 'pyears' function in the survival package in $\mathrm{R}$ (version 3.2.10). Confidence intervals for incidence rates were calculated based on the central limit theorem given dichotomous outcome in a single population. For event numbers smaller than five, agestandardised incidence rate was not reported. Using these approaches, we estimated both age-standardised and age-specific incidence rates for liver disease and incidence rates for CVD in patients with and without liver disease.

\section{Estimations of excess years life lost (YLL)}

YLL was estimated using the lillies package [25], which was validated by other studies [26-28]. YLL was estimated based on CVD onset at ages 30, 40, 50, 60, 70 and 80 (Additional file 3). We estimated excess YLL based on the specific age of onset of CVD and compared the average of these individuals to the patients without CVD of 
the same age. We also examined excess YLL by the type of first CVD presentation within age groups.

\section{Statistical analyses}

Cox proportional hazards models were fitted. Hazard ratios (HRs) from the fully adjusted models were reported with $95 \%$ confidence intervals (CI). All P values were two-sided. Models were also refitted with age group as a categorical variable to return HRs by age group. Proportional hazards assumption violations were tested for a zero slope in the scaled Schoenfeld residuals. Biomarker and BMI measurements were indicated as the presence or absence of a particular measurement above or below the stated threshold. In the primary analysis, those with missing BMI information were assumed to be non-obese. Those with missing biomarker information were assumed to be within the normal threshold on the assumption that abnormal blood test results were likely to be recorded if present. Additionally, sensitivity analyses were conducted on complete records to demonstrate that the estimates were robust to our assumptions around missing data.

Data were analysed using R (3.6.3) with the following packages: survival, tidyverse, tableone, epitools, rgdal, broom, ggplot2 and ggmap.

\section{Results}

The study cohort included 3,929,596 individuals, of whom 12,845; 2210; 1753; 3112 and 20,928 individuals were newly diagnosed with ALD, autoimmune liver disease, HBV, HCV and NAFLD, respectively (Additional file 4). The median age of diagnosis for each liver disease was ALD (54.8; IQR: 46.6-63.1), autoimmune liver disease (63.3; IQR: 53.1-72.6), HBV (47.1; IQR: 38.3-57.0), HCV (46.50; IQR: 39.3-54.9) and NAFLD (56.8; IQR: 47.8-66.6) (Additional file 4). Patient characteristics (i.e., prevalent comorbidities, biomarker values at baseline, general practice region and smoking) stratified by liver disease and sex were described in Additional file 4 . Comorbidity patterns in patients with liver disease were analysed and presented in the Additional files 1, 13.

\section{Patients with liver disease had a higher burden of CVD}

The age-standardised incidence rate for any of the five liver diseases in England was 114.5 per 100,000 person years [95\% confidence interval (CI) 112.5-116.6] (Fig. 1A, Additional file 5). For specific liver diseases, the agestandardised incidence rates per 100,000 person years were as follow: ALD (24.7, CI 23.8-25.7), autoimmune liver disease (3.7, CI 3.3-4.1), HBV (4.1, CI 3.7-4.5), HCV (6.0, CI 5.5-6.4) and NAFLD (85.5, CI 83.7-87.3) (Fig. 1A; Additional file 5). We also estimated age-specific incidence rates for liver disease and observed that the rate for NALFD peaked in individuals aged $50-59$ years
(Fig. 1B; Additional file 6). Similar trends were observed in other liver conditions of non-viral aetiology where incidence rates were as follow: ALD (age 50-59), autoimmune liver disease (age 60-69) and NAFLD (age 50-59). However, for $\mathrm{HBV}$ and $\mathrm{HCV}$, the highest incidence rates were observed in younger people, individuals aged 30-39 and individuals aged 40-49, respectively (Fig. 1B; Additional file 6).

To ascertain whether patients with liver disease had a higher incidence of CVD, we considered 17 cardiovascular conditions (see "Methods" Section). The agestandardised incidence rate for CVD in patients with any liver disease was two-fold higher than in people without liver disease. Incidence rate was 2634.6 per 100,000 person years (CI 2524.4-2744.8) in people with liver disease (Fig. 2A; Additional file 7) compared to 1339.7 per 100,000 person years (CI 1332.3-1347.0) in people without liver disease (Fig. 2B; Additional file 8). Incidence burden of CVD in patients with specific liver conditions were: ALD (3173.3; CI 2955.3-3391.3), autoimmune liver disease (2084.8; CI 1731.8-2437.8), HBV (1550.0; CI 1312.6-1787.5), HCV (1999.6; CI 1766.4-2232.7) and NAFLD (2878.0; CI 2708.1-3048.0) (Fig. 2A; Additional file 7).

The age-specific incidence rates of CVD in patients with any liver disease exhibited an upward trend with increasing age, peaking at ages 70-79 (766 per 100,000 person years). Similarly, in people without liver disease, this upward trend was maintained, albeit at a lower magnitude (highest CVD incidence was observed in individuals aged 80 and above; 562 per 100,000 person years) (Additional files 9, 10). Unlike the incidence rates of CVD which increased with age peaking in the highest age groups, the incidence rates of liver disease peaked in middle-aged individuals as shown earlier (Fig. 1B). Geographical variations of liver disease burden and CVD burden (in people with or without liver disease) were further explored and presented in the supplementary appendix.

\section{Patterns of the first presentation of CVD in patients with liver disease}

We analysed the first presentation of 17 types of incident CVD. When considering the first presentation of CVD, the number of incident CVD events for patients with liver disease were as follow; ALD (3283/12,845, 25.6\%), autoimmune liver disease $(667 / 2210,30.2 \%)$, HBV (270/1753, 15.4\%), HCV (522/3112, 16.8\%) and NAFLD (4362/20,928, 20.8\%). When comparing across patients with different liver diseases, atrial fibrillation was the most common condition while hypertrophic cardiomyopathy was the least common (Fig. 3A). When individual CVDs were grouped into five categories, coronary heart disease, which is a composite of stable angina, unstable 

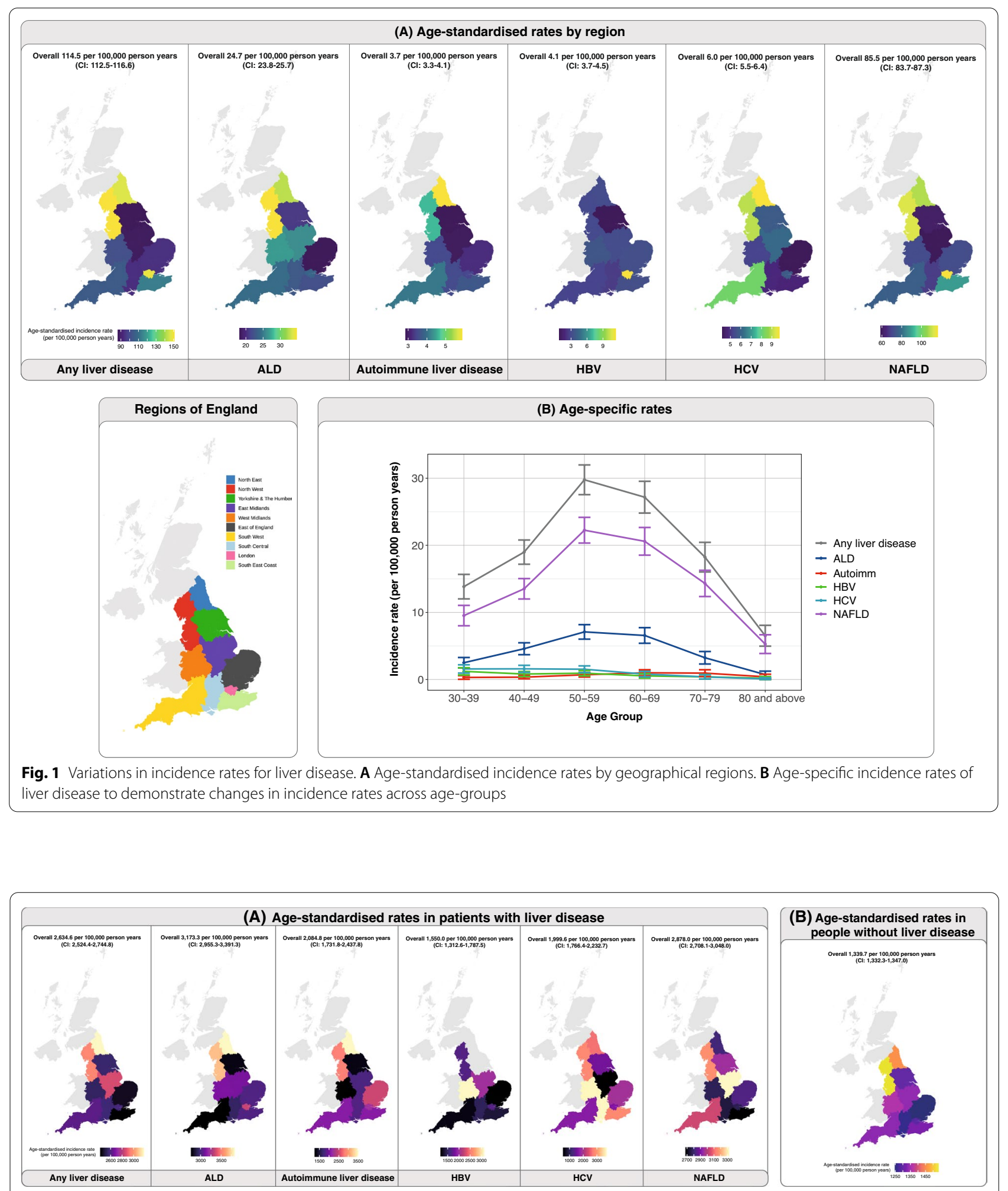

Fig. 2 Variations in incidence rates for cardiovascular disease (CVD). Age-standardised incidence rates for CVD by geographical regions in $\mathbf{A}$ patients with liver disease and $\mathbf{B}$ patients without liver disease 

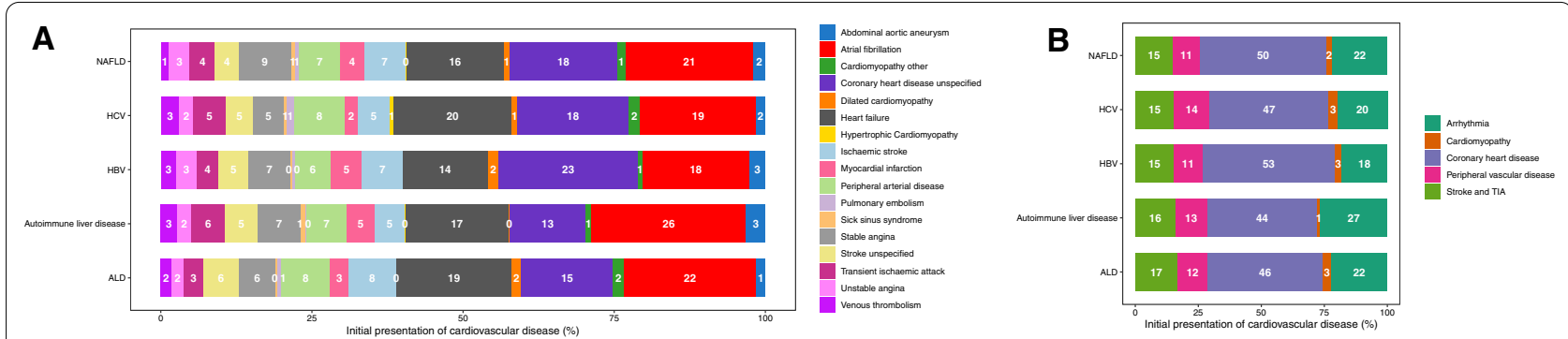

Fig. 3 Distribution of initial presentations of incident cardiovascular diseases in patients with liver disease. A Distribution of each of the 17 types of cardiovascular diseases. B Distribution by five CVD categories

angina, myocardial infarction, heart failure and coronary heart disease unspecified, was the most common, followed by arrhythmia, stroke/TIA, peripheral vascular disease and cardiomyopathy (Fig. 3B). When delving into specific liver conditions, coronary heart disease was the first presentation in $53 \%$ of patients with NAFLD who had cardiovascular events, while cardiomyopathy was the first presentation in only $3 \%$ of patients. By contrast, $44 \%$ of patients with autoimmune liver disease presenting with cardiovascular events had coronary heart disease, and $1 \%$ had cardiomyopathy (Fig. 3B).

The first presentation of CVD was influenced by age and sex (Additional file 2). CVD was more common in older individuals and there are sex differences in the type of CVD presented. In patients with $\mathrm{HCV}$, for example, $35 \%$ of men and $43 \%$ of women aged $70-79$ had a cardiovascular event (Additional file 2). In men, 5\%, 5\%, 6\% and $19 \%$ of these events account for peripheral vascular disease, stroke/TIA, arrhythmia and coronary heart disease respectively. While in women, the proportion differs with $4 \%, 15 \%$ and $24 \%$ events accounting for stroke/ TIA, arrhythmia and coronary heart disease respectively (Additional file 2).

\section{Clinical features associated with the first presentation of CVD}

The associations between patient-level factors (i.e., prevalent comorbidities, biomarkers and smoking) and the risk of incident CVD were shown in Fig. 4 and Additional file 11. We noted strong positive associations between increasing age and risk of incident CVD. Among patients with ALD, individuals aged 60-69 had a four-fold higher risk compared to 30-39-year-olds (adjusted HR: 4.98, CI 4.20-5.91). The effect of age was consistent across all liver diseases. Women had a lower risk than men; ALD (adjusted HR: 0.72, CI 0.67-0.78), autoimmune liver disease (adjusted HR: 0.53, CI 0.45-0.64), HBV (adjusted HR: 0.67, CI 0.52-0.87), HCV (adjusted HR: 0.73, CI $0.60-0.88$ ) and NAFLD (adjusted HR: 0.71, CI 0.67-0.76) (Fig. 4; Additional file 11).
We observed a consistent pattern of increased risk for a body mass index (BMI; $\mathrm{kg} / \mathrm{m}^{2}$ ) of over 30; ALD (adjusted HR: 1.20, CI 1.11-1.30), autoimmune liver disease (adjusted HR: 1.40, CI 1.17-1.67), HBV (adjusted HR: 1.50, CI 1.12-2.00), HCV (adjusted HR: 1.30, CI 1.03-1.64) and NAFLD (adjusted HR: 1.22, CI 1.15-1.30) (Fig. 4; Additional file 11). Current smoking was associated with a higher risk in patients with ALD (adjusted HR: 1.30, CI 1.18-1.43), autoimmune liver disease (adjusted HR: 1.78, CI 1.45-2.18), HBV (adjusted HR: 1.74, CI 1.25-2.43) and NAFLD (adjusted HR: 1.77, CI 1.64-1.91). The same pattern was observed in former smokers in patients with ALD, autoimmune liver disease or NAFLD.

Comorbidities such as diabetes mellitus, complications of diabetes, hypertension, proteinuric kidney disease and chronic renal disease were associated with a higher risk of CVD when comparing across all liver diseases. Diverticular disease and gastro-oesophageal reflex disease were associated with a higher risk of CVD in patients with autoimmune liver disease or NAFLD. Dyslipidaemia was also associated with a higher risk in patients with ALD (adjusted HR: 1.22, CI 1.11-1.34), HCV (adjusted HR: 1.86 , CI 1.32-2.60) or NAFLD (adjusted HR: 1.25, CI 1.17-1.34) (Fig. 4; Additional file 11).

We observed that albumin levels of $<35 \mathrm{~g} / \mathrm{L}$ were associated with a higher risk of CVD in patients with ALD (adjusted HR: 1.20, CI 1.10-1.32), autoimmune liver disease (adjusted HR: 1.55, CI 1.26-1.91), HBV (adjusted HR: 2.39, CI 1.54-3.71), HCV (adjusted HR: 1.75, CI 1.29-2.39) or NAFLD (adjusted HR: 1.59, CI 1.42-1.79). In patients with ALD or NAFLD, those who had elevated C-reactive protein levels of $\geq 10 \mathrm{mg} / \mathrm{L}$, a marker of inflammation, had at least a 1.2 -fold increased risk of CVD. Raised International Normalized Ratio (INR) of $\geq 1.7$ is associated with 3.5 -fold, 6.4-fold and 1.5-fold increased risk of CVD in patients with autoimmune liver disease, $\mathrm{HCV}$ or NAFLD. Interestingly, we noted an inverse association between alanine aminotransferase (ALT) levels and CVD risk in patients with ALD 


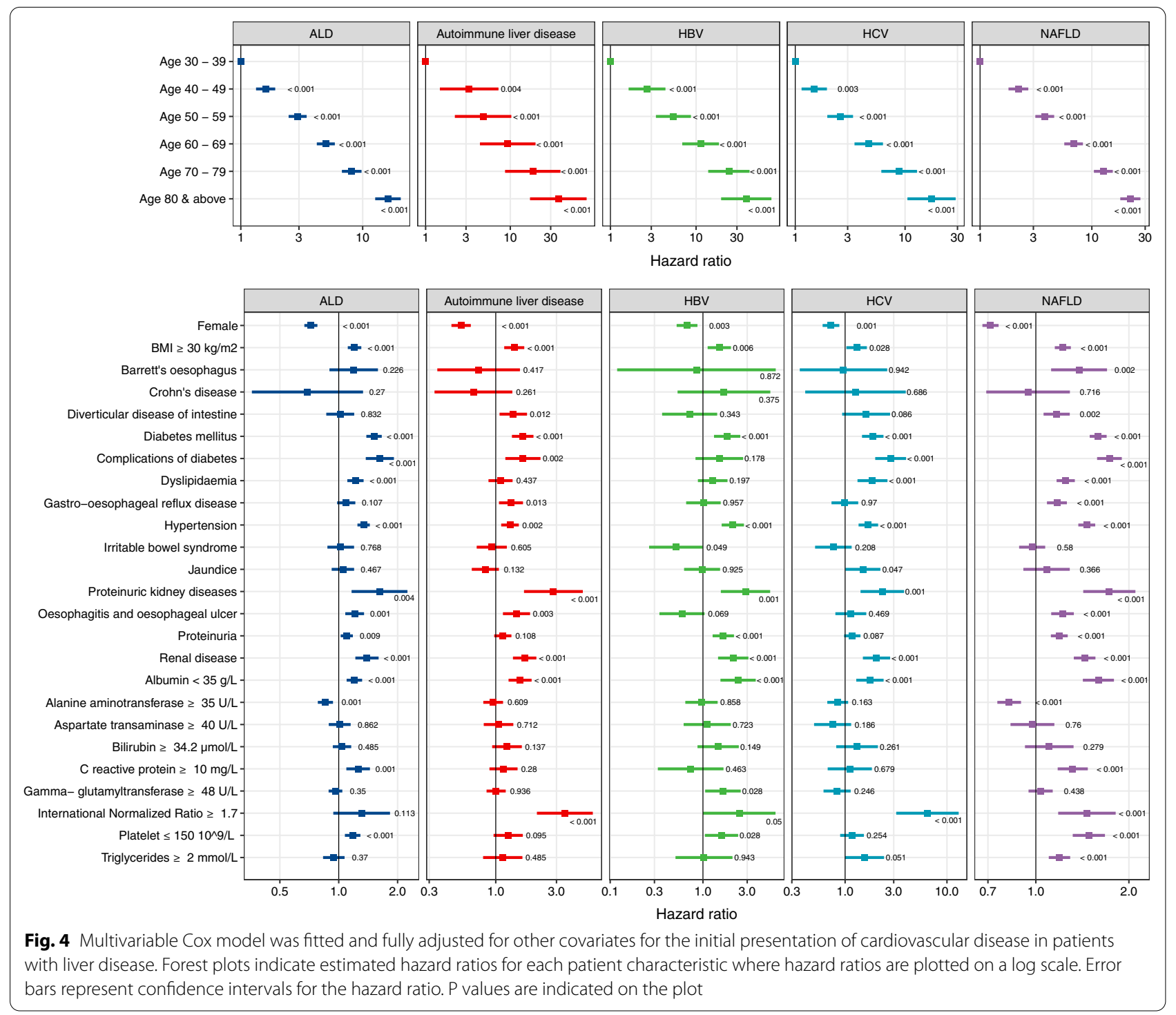

(adjusted HR: 0.85, CI 0.78-0.93) and NAFLD (adjusted HR: 0.82, CI 0.75-0.90). We performed sensitivity analyses using complete records for BMI, albumin, C-reactive protein and INR and found similar results (Additional file 12). Complete record analysis on ALT levels and CVD risk also revealed inverse associations where patients with raised ALT ( $\geq 35 \mathrm{U} / \mathrm{L}$ ) had a lower risk of CVD (reasons for this are explored in the discussion section).

\section{Excess years of life lost from CVD}

Among patients with liver disease, we estimated excess years life lost (YLL), which was calculated as the average number of years that individuals with a specific CVD condition lose in excess of that found in people without CVD of the same sex and age. We estimated excess YLL based on the age of CVD onset at ages 30, 40, 50, 60, 70 and 80 (Fig. 5A). Overall, individuals with NAFLD experienced the highest excess YLL upon CVD diagnosis, a pattern that was most pronounced at younger ages. Patients with NAFLD who developed CVD at the age of 40 years experienced an excess YLL of 18.2 years (CI 16.5-20.2) compared to those without CVD. In contrast, at the same age of CVD onset, patients with HBV experienced excess YLL of only 9.5 years (CI 7.1-12.1) (Fig. 5A).

When investigating sex differences, the difference in excess YLL between men and women appeared to be marginal in patients with ALD, autoimmune liver disease and NAFLD (Additional file 3). In patients with HBV, women diagnosed with CVD at ages 70 or 80 experience higher excess YLL than men. At age 70, women lost 


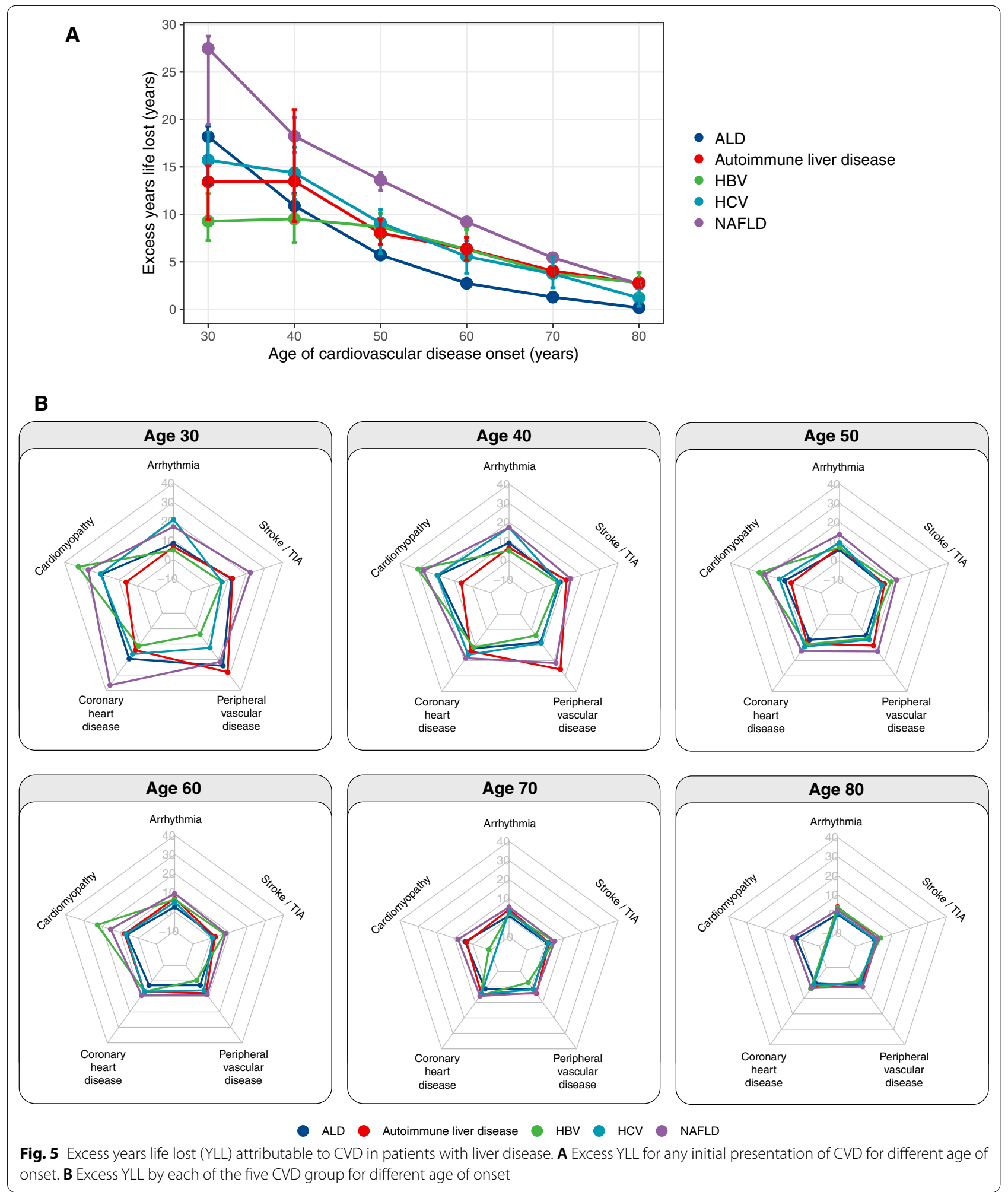

7.4 years (CI 3.2-9.0) while men lost 1.3 years $(\mathrm{CI}-0.9$ to 4.3). At age 80, women lost 6.1 years (CI 3.5-8.7) and men lost 0.2 years $(\mathrm{CI}-2.4$ to 1.0$)$ (Additional file 3 ). In patients with $\mathrm{HCV}$, women diagnosed with CVD at ages
30 and 40 experienced higher excess YLL than men. For example, at age 40 , women lost 18.6 years (CI 15.9-22.9) while men lost 12.2 years (CI 10.7-15.2) (Additional file 3). 
We also evaluated excess YLL by age of CVD onset for each of the five liver diseases stratified by the five CVD categories. In the interest of maintaining conciseness and to aid comparison across liver diseases, we graphically displayed results as radar plots (Fig. 5B). Each radar showed excess YLL estimates for a specific age of CVD onset, with each spoke representing excess YLL for one CVD category. A line is drawn to connect the excess YLL values for each CVD category where each liver disease is represented by different coloured lines. All categories of CVD were associated with shorter remaining life expectancies. When evaluating the surface areas covered by the lines, as expected, the radar plots demonstrated that younger age of CVD onset had higher excess YLL (as represented by a larger surface area connecting the CVD categories) that was consistent across the five liver conditions. Conversely, as the age of onset increased, excess YLL decreased. The radar plots also facilitate the visualisation of the differences by liver disease and CVD category. Notably, when comparing across the five liver conditions, the differences in excess YLL by CVD category were more pronounced at younger ages (Fig. 5B). At age 40, patients with autoimmune liver disease, compared to other liver diseases, experience the highest excess YLL when diagnosed with peripheral vascular disease (25.7 years; CI 12.7-41.6) compared to other CVD categories. In contrast, patients with autoimmune liver disease who are diagnosed with incident arrythmia at age 40 lost only 6.7 years $(9.5 \%$ CI $4.5-9.5)$ (Fig. 5B).

We developed a public online tool to share results interactively (https://lailab.shinyapps.io/cvd_in_liver disease/). Additional results are available in (Additional file 13).

\section{Discussion}

Our study examines incidence, comorbidity patterns, risk of initial presentation of CVD and excess YLL associated with CVD in patients with any of the five liver diseases. Patients with liver disease have an enhanced incidence burden of CVD compared to the general population. Coronary heart disease and arrhythmia are two of the most common first presentation of CVD, which show similar patterns across all liver diseases. This finding is consistent with previous studies in patients with NAFLD reporting an increase in the prevalence of coronary heart disease (including myocardial infarction), cardiac arrhythmias and cardiomyopathy [29]. Interestingly, the different aetiology of liver disease did not impact upon CVD presentation, perhaps suggesting that the liver disease drives CVD rather than the causative aetiology (i.e., viral infection or alcoholism). We provide the public, researchers and policymakers with an interactive online tool for exploration and visualisation of the incidence rates of liver disease, incidence rates of CVD in patients with and without liver disease and excess YLL associated with CVD. The tool also brings together information on other analyses such as comorbidity patterns in patients with liver disease and adjusted hazard ratios for CVD risk by age, biomarkers and comorbidities.

Our results highlight significant variations in the burden of liver disease across geographical regions in England. This suggests that there might be geographical variations in risk factors, health and risk awareness among the public, and access to diagnostic services where local solutions are required. Our observation that incidence rates for liver disease (except for HBV) were the highest in North East and North West regions was corroborated by the Public Health England's (PHE's) second atlas of variation for liver disease [30]. The PHE atlas revealed that Northern regions had a high rate of expenditure for hepatobiliary problems, liver disease admissions rates, liver disease mortality and rate of years of life lost. We observed that the incidence rate for HBV was the highest in London. This result was consistent with the PHE atlas which found that the percentage of women who tested positive for $\mathrm{HBV}$ during pregnancy screening and the rate of hospital admission for HBV-related endstage liver disease and mortality were high in London, presumably caused by a high prevalence of migrant communities in this region [30].

When examining the regional variations of CVD in patients with or without liver disease, we found that variation in incidence rates is ubiquitous across England. Our results draw attention to the need to coordinate CVD and liver disease services across regions to maintain equity in service access at a local level by helping commissioners, service providers and clinicians to compare the disease burden of their region with the national figure to ensure that care pathways are planned optimally. Regional variations signify that a one-size-fits-all approach is less likely to be effective. Services within each region need to identify challenges and adapt solutions at different rates in different scenarios. Our maps of disease burden variation could serve as a tool for benchmarking where each region stands relative to other regions and to provide a starting point for further investigation on reasons explaining the variation.

Our population-wide study of nearly 4 million individuals provides evidence that associations between clinical factors and 17 cardiovascular disease outcomes differ in terms of magnitude of effects when comparing across the five liver diseases. Raised BMI and current and former smoking were associated with an increased risk of CVD. Similarly, diabetes mellitus, hypertension, dyslipidaemia and chronic kidney disease are comorbidities associated with a higher risk. CVD 
risk is increased by at least two times in patients with impaired renal function [12]. We also observed that proteinuria, which is an indication of kidney damage, is associated with a higher risk of CVD. C-reactive protein is a marker of systemic inflammation, where it is found to be a predictor of mortality in patients with liver disease [31]. We found that patients with raised C-reactive protein have an increased risk of CVD, which corroborates previous findings that inflammation may serve as a pathogenic mechanism for triggering atherothrombotic CVD events [32]. Low albumin levels were associated with a higher risk. An inverse association between albumin and CVD may be explained by mechanisms related to the pathogenesis of CVD and inflammation associated with hypoalbuminaemia [33].

We found that markers of liver function (i.e., bilirubin, aspartate transaminase and gamma-glutamyltransferase) do not appear to be associated with CVD risk. Evidence on the association between these measurements and CVD risk has been mixed [34]. When analysing the effects of alanine aminotransferase (ALT) levels, we observed an inverse relationship between ALT and CVD risk. A systematic review and meta-analysis of 29 cohort studies demonstrated that there was limited evidence for association between ALT and CVD events [35]. In contrast, stratified analysis on cause-specific CVD endpoints demonstrated that ALT was inversely associated with coronary heart disease but positively associated with stroke [35]. A study on three large prospective cohorts (West of Scotland Coronary Prevention Study, the Prospective Study of Pravastatin in the Elderly at Risk, and the Leiden 85-plus Study) with ages ranging from 45 to 85 years, also described an inverse association between ALT and cardiovascular outcomes, even after adjusting for confounders [36]. The authors found no evidence of any association between higher levels of ALT and increased risk of clinical outcomes when examining ALT levels $>27 \mathrm{U} / \mathrm{L}$. In contrast, other studies reporting raised ALT levels with adverse outcomes were employing much higher ALT levels [37] (defined as 2 times the upper limit of normal) than in our present study (we defined raised ALT as $\geq 35 \mathrm{U} / \mathrm{L}$ ) and in the study by Ford et al. [36]. High ALT in the normal range was also found to be negatively associated with death from ischaemic heart disease [38]. This suggests that there might be potential variations of CVD risk based on ALT levels that are closer to the population median. Furthermore, another report suggested a possible U-shaped association of ALT with vascular risk [39]. Findings from our study along with others suggest that the relationship between ALT and CVD risk is more complex than currently appreciated and may be caused by differences in underlying aetiology. It is also important to note that ALT levels are not useful indicators to risk-stratify the severity of liver disease [40]. Patients with cirrhosis can have normal values when scar tissue replaces the damaged liver cells and can no longer produce ALT.

Several studies have shown that NAFLD is a risk factor for CVD, especially coronary heart disease, in a manner that is independent of other risk factors such as hypertension, diabetes, smoking and obesity [41, 42]. For example, Hamaguchi et al. found that NAFLD at baseline predisposed individuals to future CVD events, independent of typical cardiovascular risk factors [42]. All subtypes of NAFLD were linked to increased CVD risk, but individuals with fibrosis and NASH have an even higher risk [41]. The pathophysiological mechanisms of NAFLD and CVD have been elegantly reviewed by Przybyszewski et al. recently [43]. Although there is a lack of complete understanding of the exact causal mechanisms, it was thought that inflammation and dyslipidemia may promote atherogenesis leading to coronary heart disease [43]. During NAFLD progression, inflammation of visceral adipose tissue may trigger the activation of proinflammatory pathways (JNK and NF- $\mathrm{kB}$ ) and downstream synthesis of procoagulant factors, collectively causing increased CVD risk [43].

Our results confirm that patients with liver disease experience premature mortality attributable to CVD. These findings are consistent with a report from the Centers for Disease Control and Prevention stating that CVD ranks third in YLL prior to age 65 [44] along with other studies demonstrating that premature death due to CVD continues to increase globally [45, 46]. Although each type of CVD is associated with excess YLL, we observed that women lost more years of life compared to men at a younger age of CVD onset among patients with $\mathrm{HCV}$ and at an older age of CVD onset among patients with HBV.

\section{Strengths and weaknesses}

A major strength of this study is the ability to differentiate 17 cardiovascular outcomes using a real-world population cohort, which is larger, contemporary and more representative of the general population compared with investigator-led cohorts. Given that we have analysed records from both primary and secondary care, we are able to capture conditions that are treated by general practitioners and specialist clinicians in hospitals. Primary care records also provide more detailed data than those recorded on admission to hospital, where the former account for the total population while the latter only for a subset of individuals who attended hospitals. In addition, given the longevity of follow-up and the breadth and depth of variables available, we were able to examine the associations between a wide range of comorbidities 
and biomarkers. Another strength is our use of openly accessible codelists for reuse that are made available publicly (https://phenotypes.healthdatagateway.org/home/) and previously validated in numerous studies $[18,19,21]$.

We note important limitations in this study. First, our study employs only data in England, which may limit generalisability to other geographical locations. We have only sampled a subset of English general practices accounting for almost 4 million individuals. Second, as in all observational studies, there is the potential for residual confounding. Third, missing data is a common phenomenon in EHRs, however, sensitivity analyses on complete records have shown that our estimates were robust to our assumptions around missing data. There remains a possibility of underreporting of NAFLD in the nineties and early 2000s.

\section{Policy implications}

Our study has policy implications in CVD prevention and targeted recommendations. CVD prevention policies and guidelines should identify groups of high-risk individuals among people with liver disease for targeted screening and intervention. Treatment of $\mathrm{HCV}$ with direct-acting antiviral therapy is linked to a decrease in the risk of CVD events in patients who have sustained virological responses [47]. This suggests that $\mathrm{HCV}$ infection and potentially HBV infection may be modifiable CVD risk factors [48] and treating the underlying infection could improve both liver and CVD outcomes.

This work has important implications for addressing inequalities in HBV and HCV screening and treatment. According to recent estimates, around 1,13,000 and $1,80,000$ individuals are infected with $\mathrm{HCV}$ and $\mathrm{HBV}$ in England respectively [49]. Although effective treatment for $\mathrm{HCV}$ exists, there remains inequality in accessing treatment as HCV may remain undiagnosed in underserved populations such as people who are homeless, in prison or drug users. Migration from HBV endemic countries has resulted in an increased prevalence of HBV infection in the UK. However, migrants remained an underserved or invisible population due to low disease awareness and low engagement with health services [50]. A study investigating the uptake of HBV testing in England revealed that there were low awareness levels of HBV among the migrant community, which resulted in limited engagement with healthcare services [50]. Furthermore, HBV infection is a stigmatised condition, which further exacerbated the testing and diagnostic barriers. HBV infection in the UK is thought to be an invisible disease within an invisible population' [50]. These observations were corroborated in another study which found limited evidence of HBV testing among migrants in the UK and testing in children and young people remain very low [51]. Our results suggest that regional initiatives targeting underserved communities might help complement interventions from two perspectivestreatment of chronic $\mathrm{HBV} / \mathrm{HCV}$ by addressing social challenges that cause healthcare inequality and targeted prevention of CVD in patients with liver disease.

\section{Conclusion}

We propose that management of liver disease should include regular cardiovascular risk assessment in addition to checking for undiagnosed diabetes and renal comorbidities. Furthermore, CVD risk assessment should be provided for all types and all stages of liver disease. At the individual level, communicating risk information may affect people's attitude to seek opportunities for early detection and treatment of CVD to reduce premature mortality. For example, this can be accomplished by improving access to the NHS Health Check programme [52], aimed at individuals aged 40-74, to identify early signs of CVD, stroke, kidney disease and diabetes. Access to risk information could help stimulate conversations between patients and their doctors on decisions on managing their health and care. It is now possible to generate and disseminate novel risk information using real-world data that takes a step towards making decisions that are not based on the group average but that are closer to specific patient scenarios (i.e., patients like me) encountered in clinical settings [53].

\section{Abbreviations}

CVD: Cardiovascular disease; HER: Electronic health records; ALD: Alcoholic liver disease; HBV: Chronic hepatitis B infection; HCV: Chronic hepatitis C infection; NAFLD: Non-alcoholic fatty liver disease; YLL: Years of life lost; INR: International normalised ratio; TIA: Transient ischaemic attack; HR: Hazard ratio; $\mathrm{Cl}$ : Confidence interval; ALT: Alanine aminotransferase.

\section{Supplementary Information}

The online version contains supplementary material available at https://doi. org/10.1186/s12967-021-03210-9.

Additional file 1: Comorbidity patterns in patients with liver disease separated by sex and age.

Additional file 2: Proportion of patients within each age-sex stratum experiencing incident CVD (first presentation) are shown for $\mathbf{A}$ each of the 17 individual CVDs. B The 17 conditions are grouped into five categories and proportions are shown for each category.

Additional file 3: Excess years life lost (YLL) attributable to CVD in patients with liver disease separated by sex.

Additional file 4: Baseline characteristics for patients with liver disease.

Additional file 5: Age-standardised incidence rates for liver disease.

Additional file 6: Age-specific incidence rates for liver disease.

Additional file 7: Age-standardised incidence rates for cardiovascular disease in patients with liver disease. 
Additional file 8: Age-standardised incidence rates for cardiovascular disease in patients without liver disease.

Additional file 9: Age-specific incidence rates for cardiovascular disease in patients with liver disease.

Additional file 10: Age-specific incidence rates for cardiovascular disease in patients without liver disease.

Additional file 11: Factors associated with the initial presentation of cardiovascular disease in individuals with liver disease.

Additional file 12: Sensitivity analyses on complete records.

Additional file 13. Supplementary methods and results.

\section{Acknowledgements}

Not applicable.

\section{Authors' contributions}

Research question: WHC and AGL. Funding: AGL. Study design and analysis plan: WHC, SM and AGL. Preparation of data: WHC, SM and AGL. Statistical analysis: WHC, SM and AGL. Creation of online app: SM. Drafting initial and final versions of manuscript:WHC and AGL. Critical review of early and final versions of manuscript: All authors. All authors read and approved the final manuscript.

\section{Funding}

AGL is supported by funding from the Wellcome Trust (204841/Z/16/Z), National Institute for Health Research (NIHR) University College London Hospitals Biomedical Research Centre (BRC714/HI/RW/101440), the Academy of Medical Sciences (SBF006\1084), NIHR Great Ormond Street Hospital Biomedical Research Centre (19RX02) and the Health Data Research UK Better Care Catalyst Award (CFC0125). The funders had no role in study design, data collection and analysis, decision to publish, or preparation of the manuscript.

\section{Availability of data and materials}

The data used in this study are available on successful ethics application to the Clinical Practice Research Datalink (CPRD). All summarised data and results are made available as Additional files.

\section{Declarations}

\section{Ethics approval and consent to participate}

Information governance approval was obtained from the Medicines Healthcare Regulatory Authority (UK) Independent Scientific Advisory Committee (21_000363) Clinical Practice Research Datalink (CPRD).

\section{Consent for publication}

Not applicable.

\section{Competing interests}

GRF receives funding from companies that manufacture drugs for hepatitis C virus (AbbVie, Gilead, MSD) and consults for GSK, Arbutus and Shionogi in areas unrelated to this research.

\section{Author details}

${ }^{1}$ Institute of Health Informatics, University College London, London, UK. ${ }^{2}$ Barts Liver Centre, Blizard Institute, Queen Mary University of London, London, UK.

Received: 16 September 2021 Accepted: 19 December 2021

Published online: 03 January 2022

\section{References}

1. NICE. Cardiovascular disease: risk assessment and reduction, including lipid modification. https://www.nice.org.uk/guidance/cg181. Accessed 19 Mar 2021.
2. NICE. Cardiovascular disease prevention. https://www.nice.org.uk/guida nce/ph25/chapter/2-Public-health-need-and-practice. Accessed 19 Mar 2021.

3. Alexander M, Loomis AK, Van Der Lei J, et al. Non-alcoholic fatty liver disease and risk of incident acute myocardial infarction and stroke: findings from matched cohort study of 18 million European adults. BMJ. 2019;367:1-9.

4. Younossi ZM, Koenig AB, Abdelatif D, Fazel Y, Henry L, Wymer M. Global epidemiology of nonalcoholic fatty liver disease - meta-analytic assessment of prevalence, incidence, and outcomes. Hepatology. 2016;64:73-84.

5. The LPH. Tackling obesity seriously: the time has come. Lancet Public Health. 2018;3:e153.

6. World Health Organisation. Hepatitis B. https://www.who.int/news-room/ fact-sheets/detail/hepatitis-b. Accessed 19 Mar 2021.

7. World Health Organisation. Hepatitis C. https://www.who.int/newsroom/fact-sheets/detail/hepatitis-c. Accessed 19 Mar 2021.

8. Lee KK, Stelzle D, Bing R, et al. Global burden of atherosclerotic cardiovascular disease in people with hepatitis C virus infection: a systematic review, meta-analysis, and modelling study. Lancet Gastroenterol Hepatol. 2019;4:794-804

9. Babiker A, Jeudy J, Kligerman S, Khambaty M, Shah A, Bagchi S. Risk of cardiovascular disease due to chronic hepatitis $C$ infection: a review. J Clin Transl Hepatol. 2017;5:343.

10. White DL, Ratziu V, El-Serag HB. Hepatitis C infection and risk of diabetes: a systematic review and meta-analysis. J Hepatol. 2008;49:831-44.

11. Wong F. Renal diseases and the liver. Clin Liver Dis. 2011;15:39-53.

12. Gansevoort RT, Correa-Rotter R, Hemmelgarn BR, et al. Chronic kidney disease and cardiovascular risk: epidemiology, mechanisms, and prevention. Lancet. 2013;382:339-52.

13. Chalasani N, Younossi Z, Lavine JE, et al. The diagnosis and management of nonalcoholic fatty liver disease: practice guidance from the American Association for the Study of Liver Diseases. Hepatology. 2018;67:328-57.

14. European Association for the Study of The Liver EA, European Association for the Study of Diabetes. EASL-EASD-EASO Clinical Practice Guidelines for the management of non-alcoholic fatty liver disease. Obes Facts. 2016;9:65-90.

15. Williams R, Aspinall R, Bellis M, et al. Addressing liver disease in the UK: a blueprint for attaining excellence in health care and reducing premature mortality from lifestyle issues of excess consumption of alcohol, obesity, and viral hepatitis. Lancet. 2014;384:1953-97.

16. Williams $R$, Ashton $K$, Aspinall $R$, et al. Implementation of the Lancet Standing Commission on liver disease in the UK. Lancet. 2015;386:2098-111.

17. Williams R, Alexander G, Aspinall R, et al. New metrics for the Lancet Standing Commission on liver disease in the UK. Lancet. 2017;389:2053-80

18. Kuan V, Denaxas S, Gonzalez-Izquierdo A, et al. A chronological map of 308 physical and mental health conditions from 4 million individuals in the English National Health Service. Lancet Digit Heal. 2019;1:e63-77.

19 Denaxas S, Gonzalez-Izquierdo A, Direk K, et al. UK phenomics platform for developing and validating electronic health record phenotypes: CALIBER. J Am Med Informatics Assoc. 2019. https://doi.org/10.1093/ jamia/ocz105.

20 Pasea L, Chung S-C, Pujades-Rodriguez M, et al. Bleeding in cardiac patients prescribed antithrombotic drugs: electronic health record phenotyping algorithms, incidence, trends and prognosis: Supplementary Appendix. BMC Med. 2019. https://doi.org/10.1186/s12916-019-1438-y.

21 Lai AG, Pasea L, Banerjee A, et al. Estimated impact of the COVID-19 pandemic on cancer services and excess 1-year mortality in people with cancer and multimorbidity: near real-time data on cancer care, cancer deaths and a population-based cohort study. BMJ Open. 2020. https:// doi.org/10.1136/bmjopen-2020-043828.

22. Rapsomaniki E, Timmis A, George J, et al. Blood pressure and incidence of twelve cardiovascular diseases: lifetime risks, healthy life-years lost, and age-specific associations in 1.25 million people. Lancet. 2014;383:1899-911.

23. Shah AD, Langenberg C, Rapsomaniki E, et al. Type 2 diabetes and incidence of cardiovascular diseases: a cohort study in 1.9 million people. Lancet Diabetes Endocrinol. 2015;3:105-13. 
24. Office for National Statistics. Estimates of the population for the UK, England and Wales, Scotland and Northern Ireland. https://www.ons.gov.uk/ peoplepopulationandcommunity/populationandmigration/population estimates/datasets/populationestimatesforukenglandandwalesscotla ndandnorthernireland. Accessed 23 Apr 2020.

25. Plana-Ripoll O, Canudas-Romo V, Weye N, Laursen TM, McGrath JJ, Andersen PK. lillies: an R package for the estimation of excess life years lost among patients with a given disease or condition. PLoS ONE. 2020;15:e0228073.

26. Erlangsen A, Andersen PK, Toender A, Laursen TM, Nordentoft M, Canudas-Romo V. Cause-specific life-years lost in people with mental disorders: a nationwide, register-based cohort study. Lancet Psychiatry. 2017:4:937-45.

27. Plana-Ripoll O, Pedersen CB, Agerbo E, et al. A comprehensive analysis of mortality-related health metrics associated with mental disorders: a nationwide, register-based cohort study. Lancet. 2019;394:1827-35.

28. Laursen TM, Plana-Ripoll O, Andersen PK, et al. Cause-specific life years lost among persons diagnosed with schizophrenia: is it getting better or worse? Schizophr Res. 2019;206:284-90.

29. Kasper P, Martin A, Lang S, et al. NAFLD and cardiovascular diseases: a clinical review. Clin Res Cardiol. 2020. https://doi.org/10.1007/ s00392-020-01709-7.

30. Public Health England. Public Health England. The 2nd Atlas of variation in risk factors and healthcare for liver disease in England. https://finge rtips.phe.org.uk/profile/atlas-of-variation. 2017;12-30. Accessed $16 \mathrm{Apr}$ 2021.

31. Cervoni J-P, Thévenot T, Weil D, et al. C-reactive protein predicts shortterm mortality in patients with cirrhosis. J Hepatol. 2012;56:1299-304.

32. Bisoendial RJ, Boekholdt SM, Vergeer M, Stroes ESG, Kastelein JJP. C-reactive protein is a mediator of cardiovascular disease. Eur Heart J. 2010;31:2087-91.

33. Ronit A, Kirkegaard-Klitbo DM, Dohlmann TL, et al. Plasma albumin and incident cardiovascular disease: results from the CGPS and an updated meta-analysis. Arterioscler Thromb Vasc Biol. 2020;40:473-82.

34. Targher G, Byrne CD. Circulating markers of liver function and cardiovascular disease risk. Arterioscler Thromb Vasc Biol. 2015;35:2290-6.

35. Kunutsor SK, Apekey TA, Khan H. Liver enzymes and risk of cardiovascular disease in the general population: a meta-analysis of prospective cohort studies. Atherosclerosis. 2014;236:7-17.

36. Ford I, Mooijaart SP, Lloyd S, et al. The inverse relationship between alanine aminotransferase in the normal range and adverse cardiovascular and non-cardiovascular outcomes. Int J Epidemiol. 2011;40:1530-8.

37. Lee TH, Kim WR, Benson JT, Therneau TM, Melton LJ III. Serum aminotransferase activity and mortality risk in a United States community. Hepatology. 2008;47:880-7.

38. Schooling CM, Kelvin EA, Jones HE. Alanine transaminase has opposite associations with death from diabetes and ischemic heart disease in NHANES III. Ann Epidemiol. 2012;22:789-98.

39. Schindhelm RK, Dekker JM, Nijpels G, et al. Alanine aminotransferase predicts coronary heart disease events: a 10-year follow-up of the Hoorn Study. Atherosclerosis. 2007;191:391-6.

40. Lominadze Z, Kallwitz ER. Misconception: you can't have liver disease with normal liver chemistries. Clin liver Dis. 2018;12:96.

41. Targher G, Byrne CD, Lonardo A, Zoppini G, Barbui C. Non-alcoholic fatty liver disease and risk of incident cardiovascular disease: a meta-analysis. J Hepatol. 2016;65:589-600.

42. Hamaguchi M, Kojima T, Takeda N, et al. Nonalcoholic fatty liver disease is a novel predictor of cardiovascular disease. World J Gastroenterol WJG. 2007;13:1579.

43. Przybyszewski EM, Targher G, Roden M, Corey KE. Nonalcoholic fatty liver disease and cardiovascular disease. Clin Liver Dis. 2021;17:19-22.

44. Centers for Disease Control and Prevention. Years of life lost from cardiovascular disease. https://www.cdc.gov/mmwr/preview/mmwrhtml/ 00000809.htm. Accessed 19 Mar 2021.

45. Roth GA, Mensah GA, Johnson CO, et al. Global burden of cardiovascular diseases and risk factors, 1990-2019: update from the GBD 2019 study. J Am Coll Cardiol. 2020;76:2982-3021.

46. Iyer DG, Shah NS, Hastings KG, et al. Years of potential life lost because of cardiovascular disease in Asian-American subgroups, 2003-2012. J Am Heart Assoc. 2019;8:e010744.
47. Butt AA, Yan P, Shuaib A, Abou-Samra A-B, Shaikh OS, Freiberg MS. Directacting antiviral therapy for HCV infection is associated with a reduced risk of cardiovascular disease events. Gastroenterology. 2019;156:987-96.

48. Cacoub P. Hepatitis $C$ virus infection, a new modifiable cardiovascular risk factor. Gastroenterology. 2019;156:862-4.

49. Public Health England. Tackling inequalities in hepatitis $C$ testing and treatment. https://publichealthmatters.blog.gov.uk/2019/07/28/tacklinginequalities-in-hepatitis-c-testing-and-treatment/. Accessed 19 Mar 2021.

50. Lee ACK, Vedio A, Liu EZH, Horsley J, Jesurasa A, Salway S. Determinants of uptake of hepatitis B testing and healthcare access by migrant Chinese in the England: a qualitative study. BMC Public Health. 2017;17:1-11.

51. Evlampidou I, Hickman M, Irish C, et al. Low hepatitis B testing among migrants: a cross-sectional study in a UK city. Br J Gen Pract. 2016:66:e382-91.

52. NHS Health Check. https://www.nhs.uk/conditions/nhs-health-check/. Accessed 19 Mar 2021.

53. Lai AG, Chang WH, Parisinos CA, Katsoulis M, Blackburn RM, Shah AD, Nguyen V, Denaxas S, Davey Smith G, Gaunt TR, Nirantharakumar K, Cox MP, Forde D, Asselbergs FW, Harris S, Richardson S, Sofat R, Dobson RJB, Hingorani A, Patel R, Sterne J, Banerjee A, Denniston AK, Ball S, Sebire NJ, Shah NH, Foster GR, Williams B, Hemingway H. An informatics consult approach for generating clinical evidence for treatment decisions. BMC Med Inform Decis Mak. 2021;21(1):281. https://doi.org/10.1186/ s12911-021-01638-z.

\section{Publisher's Note}

Springer Nature remains neutral with regard to jurisdictional claims in published maps and institutional affiliations.

Ready to submit your research? Choose BMC and benefit from

- fast, convenient online submission

- thorough peer review by experienced researchers in your field

- rapid publication on acceptance

- support for research data, including large and complex data types

- gold Open Access which fosters wider collaboration and increased citations

- maximum visibility for your research: over $100 \mathrm{M}$ website views per year

At BMC, research is always in progress.

Learn more biomedcentral.com/submissions 